\title{
Highly Cesium Selective Calix[6]arene Receptors: Synthesis, Structure and Cesium Binding Properties of Calix[6]arene Biscrown
}

\author{
Seung Whan Ko, Yong Shik Yang, Jung Hwa Mun, Ki-Min Park, ${ }^{\dagger}$ Shim Sung Lee, and Kye Chun Nam" \\ Department of Chemisty and Institute of Basic Science, Chomam Kational Linersity, (indangit 500-757, Korea

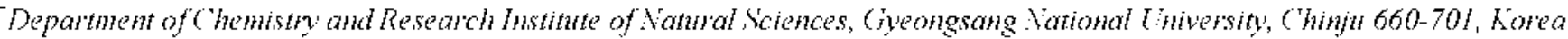 \\ Received Juh 30,2002
}

Key Words : Caliv[6]arene, Biscronn, Cesium selective reesptor

Cesium- 137 is a relatively abundant nuclear fission product and constitutes a major sounce of heat in nuclear wastes..2 along with strontium-90. Much effort has been made to the development of improved processes ${ }^{3.4}$ for the removal of cesium- 137 from nuclear wastes. For the efficient removal of cesium. several crown ethers have been prepared and their binding properties were investigated. For the development of a selective ligand for cesium ion calix[6]arenes have been utilized as a receptor. When six ester groups were introduced at the lower rim. a ligh binding property for cesium ion was reported. ${ }^{5}$ But selectivity over potassium and rubidium is not quite ligh. The lack of selectivity has been attributed into the flexibility of the parent calix[6]arene moieties. The most effective approach to make them inmobile is to build a bridge at the lower. Since Gutscheis lower rim-bridged calix[6]arene ${ }^{\text {ri }}$ in 1993. several lower rim-multibridged calix[6]arenes and their ion binding properties were reported ${ }^{7-9}$ Recently. calix[6]arene bis-crown-4 was synthesized and it showed a high selectivity for cesium. ${ }^{11}$ For the purpose of developing cesium selective ion receptors, we prepared three new calix[6]arene biscrowns and in estigated their structural and metal binding properties.

ln order to increase ion binding strength. two methosymethyl substituents $\left(-\mathrm{CH}_{2} \mathrm{OCH}_{3}\right)$ at the l.4-position of calix[+]arene are introduced by treating calix[6]arene with methoxymethyl chloride in the presence of $\left(\mathrm{CH}_{3}\right)_{5} \mathrm{SiOK} . "$ Benzil derivative was also prepared for comparison. A mixture of calix[6]arene biscrown 2a and 3a were synthesized when 1a was treated with tri-ethy lene glycol ditosy late in the presence of $\mathrm{Cs}_{2} \mathrm{CO}_{3}$ in benzene as shown in Figure $\mathrm{I}$. The total amount of two conformers was consistently about $35 \%$ of the reaction mixture and the two isomers were isolated by column chromatography. Two conformers can be identified from ${ }^{1} \mathrm{H}$ NMR spectra. ${ }^{8}$ The cone conformation was clearly demonstrated by presence of two $A B$ quartets for the $\mathrm{ArCH}_{2} \mathrm{Ar}$ protons $\left(2: 1\right.$ ratio) in the ${ }^{1} \mathrm{H}$ NMR spectrum at $\delta$ 4.6.3.6 and 3.t. On the other hand. the methylene protons of 1.2.3-altemate conformation appeared as an $\mathrm{AB}$ quartet for sin-oriented aromatic rings and a singlet for antioriented rings. But only cone conformer $\mathbf{2 b}$ was obtained when benzyl derivative 1 b treated under the same condition. indicating that substituents R could play an important role for the formation of 1.2.3-alternate conformer. Free hydroxy calix[6]arene biscrown + is obtained when $R$ groups are

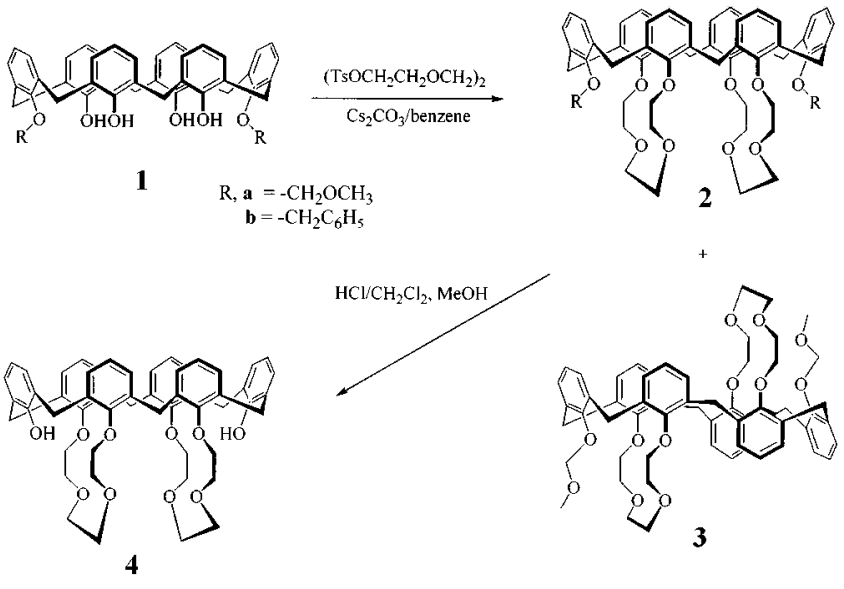

Figure 1. Synthesis of calix[6]arene-bis-crown-4.

removed by treating 2a and $3 \mathbf{a}$ with $\mathrm{HCl}$ in $\mathrm{CH}_{2} \mathrm{Cl}_{2}$ and $\mathrm{MeOH}$. indicating that the fixed 1.2.3-alternate and cone conformers could exist only when the large $\mathrm{R}$ groups were present to prevent the rotation.

Figure 2 showed a X-ray structure of 1.2.3-altemate calix[6]arene biscrown 3a. Two crown units as well as two methoxymethyl groups stayed at the opposite side of calixarene circle. indicating that the inversion point existed

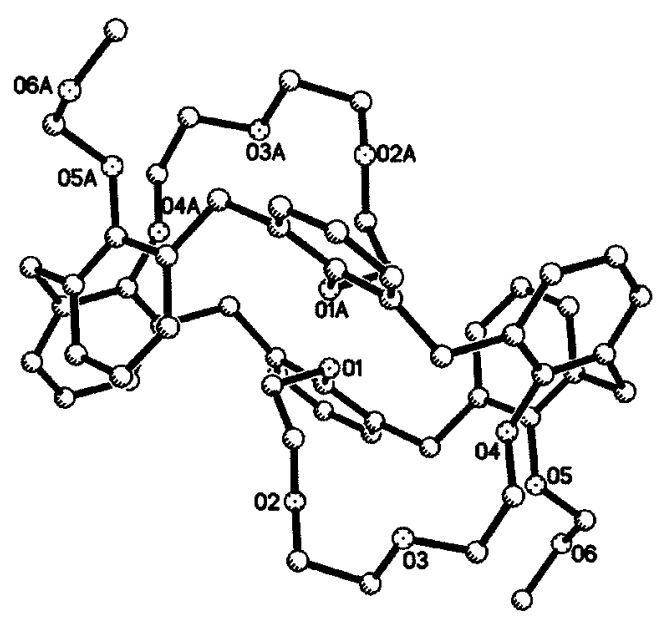

Figure 2. Crystal structure of 3a. Solvent molecules tour molecules of benzenc) and hydrogen atoms hase been omitted for clarily: 
Table 1. Cristal dala and structure refunemennt for 3a

\begin{tabular}{|c|c|}
\hline Jimpirical formula & $660) 160 \mathrm{C}^{\prime} 16012$ \\
\hline Crỵtul sỵstem & Iriclinic \\
\hline Space group & $P-1$ \\
\hline Unit cell dimensions & $\begin{array}{l}a-10.2536(13) \wedge \alpha-102.215(3)^{\prime \prime} \\
b-13.0158(16) \wedge \beta-92.621(3)^{\circ} \\
c-23.641(3) \wedge \gamma-114.494(3)^{\circ}\end{array}$ \\
\hline Relimement method & l'ull-muatrix least-squares on $\mathrm{I}^{2}$ \\
\hline Goodness-ol-1it ou $1: 2$ & $0.9] 1$ \\
\hline lijnal $R$ indices [I - 2sigman(l)] & $R 1-0.0880$. . R2 $2-0.1873$ \\
\hline$R$ indices (all data) & $R]-0.3317 . w R 2-11.28601$ \\
\hline I.argest difl. peak and hole & 0.343 and -0.354 e. $\AA^{-3}$ \\
\hline
\end{tabular}

Table 2. Percentage extraction (\%) of alkali picrates from water into $\mathrm{C}_{2} \mathrm{Cl}_{2}$ at $25^{\circ} \mathrm{C}^{\circ}$

\begin{tabular}{cccccc}
\hline & \multicolumn{5}{c}{${ }^{{ }^{a} a l i}$} \\
\cline { 2 - 6 } & $\mathrm{J}, j$ & $\mathrm{Na}^{\prime}$ & $\mathrm{K}$ & $\mathrm{Rb}$ & $\mathrm{Cs}$ \\
\hline $2 \mathbf{a}$ & 0 & 0 & 0 & 3 & 57 \\
4 & 0 & 0 & 0 & 4 & 33 \\
\hline & 0 & 0 & 0 & 3 & 11 \\
\hline
\end{tabular}

${ }^{2} \mathrm{I} \times 10^{-1} \mathrm{M}$ receptor solution in $\mathrm{CII}_{2} \mathrm{Cl}_{2}$ was shaken ( I lor) with $1 \times 10^{-1}$ $M$ picrate salt solution in $\mathrm{H}_{2} \mathrm{O}$ and the percentage exitraction was measured from the resulting absorbance at $355 \mathrm{~nm}$.

at the center of calixarene framework. It is believed that this is the first example of the X-ray structure of 1.2.3-conformer of calix[6]arene so far. Table 1 showed the detail data for Xray stnicture 3:a

The alkali metal binding properties were investigated from two plases extraction experiment where aqueous solutions of the picrate salts are shaken with methylene chloride solutions of ligands. The amount of metal ion was determined from picrate absorption in the UV spectrum. Table 2 showed the percent extraction of metal picrate salts by $\mathbf{2 a}$. 2b. 3a and 4. 1.2.3-Alternate conformer 3a showed no metal extraction at all. The cone conformer 2 a extracted $57 \%$ of the cesium picrate from aqueous solution into the methy lene clloride solution of the host at a $1: 1$ ratio. but only $3 \%$ of the rubidium and almost none of the others such as $\mathrm{Li}^{\prime}$. $\mathrm{Na}$ ' and $\mathrm{K}$ salts. Extraction percentage of cesium salt decreased to $33 \%$ for $\mathbf{2 b}$. indicating that the large benzyl groups could inlibit the cesium binding from two crown units. Further decrease of cesium extraction to $11 \%$ was observed when free hydroxy groups are present with host 4 . indicating that free $\mathrm{OH}$ groups could compete the alkali metal binding for ligand as expected. ISE experiments are in progress using PVC membrane electrodes.

Acknow ledgment. This work was supported by Grant No. R01-2000-00047 from the Basic Research Program of the Korea Science \& Engineering Foundation. NMR spectra were taken at the Korea Basic Science Institute. Gwangju. Korea.

\section{Refereneces and Notes}

1. Ancl. Haste Nerws 1993, 13. 453.

2. Stedwell. M. I.: Buns. R. E. ('hem. Fong. Prog. 1957. 53.93,

3. Enest. M. V.: Bibler. J. '?.: Whilley. R. D.: Lindat Wanc. N. H. Ihd. Fng. (hem. Rev 1997. 36. 2775.

4. Mimura, II.: I ehto. J.: I Iarjula, R. J. Anc. Sci. Tkch. 1997. 34, 607.

5. Amaud-Neu, F.: Collins. F.. M.: Deasy, M.: Ferguson, C.: H Larris, S. J: Kaitner, B.: I.ough. A. J: MeKerver: M. A.: Marques. F.: Ruhl. B. L.: Schwing-Weill. M. J.: Seward. E. M. J. Am. Chem. Soc. 1989. 111.8681,

6. Kanamathareddy. S.: Gutsche. C. D.J.Lm Chom. Soc. 1993. 115. 6572.

7. Chen, Y: I.i, I.: Xitl. J.: 7.hong, 7.: Gong. S.: I.u, X. Smh. Commmin. 1999. 29.705.

8. Otsuka. H.: Araki. K.: Malsumoto. H.: Harada. [.: Shinlai. \$. J. Org. (hem, 1995. 60. 4862 .

9. Otsuka. H.: Suzuki. Y.: Ikeda. A.: Arahi. K.: Shinkai. S. Tetrathedron 1998. 54. 423.

10. Blanda, M. T.: Farmer, D. B.: Brodbelt. J. S.: Goolsby. B. I. J. Am. Chem. Soc. 2000. 122, 1486.

11. Nam. K. C.: Ko. S. W.: Kang. S. O.: Lee. S. H.: Kim. D. S. J. Incl. Phenom. 2001. f0. 285 .

12. Some representative spectroscopic data for 2 a. 2 b. 3a and $\downarrow$ are as follows. 2a: mp-238-241 "C: 'H NMR (CDCl $) 87.59-7.57$ (d ot d, $4 \mathrm{II}, \wedge \mathrm{rH}, J-6.69 \mathrm{HI}$ ). 7.1.3-7.09 (d of t. 8II. $\wedge \mathrm{rII}), 6.31$ (t. $2 \mathrm{II}$, $\Lambda \mathrm{rI}, J-7.68 \mathrm{HI}$ ). 6.05 (d. $4 \mathrm{H}$. $\Lambda \mathrm{rII}, J-7.68 \mathrm{H}$ ). 5.14 (s. $+\mathrm{H}$. $-\left(\mathrm{CH}_{2}-\right) .4 .61$ (d. $2 \mathrm{H}$. ArCH $\mathrm{CH}_{2} \mathrm{Ar} J-13.23 \mathrm{~Hz}$ ). 4.60 (d. $4 \mathrm{H}$. $\left.\mathrm{ArCH}_{2} \mathrm{Ar} . J-16.29 \mathrm{HL}\right) .4 .03$ (t. $4 \mathrm{H} .-\left(\mathrm{CCH}_{2} \mathrm{CH}_{2} \mathrm{O}-. . J-9.09 \mathrm{~Hz}\right)$. $3.83-3.73$ (m. 12H. $-\mathrm{OCH}_{2} \mathrm{CH}_{2} \mathrm{O}$ )- 3.71 (s. $6 \mathrm{H}$. $\left.-\mathrm{OCH}_{3}\right) .3 .62(\mathrm{~d}$. $4 \mathrm{II}, \mathrm{ArCII} \wedge \mathrm{r}, J-16.29 \mathrm{II}$ ). $3.57-3.39$ (m. $6 \mathrm{II}, \wedge \mathrm{rCH} \mathrm{I}_{2} \wedge \mathrm{r}$ and $-\mathrm{OCI}_{2} \mathrm{CII}_{2} \mathrm{O}$-). 3.13 (d, $4 \mathrm{II}_{2}-\mathrm{OCII}_{2} \mathrm{CII}_{2} \mathrm{O}-, J-10.74 \mathrm{II}$.) ${ }^{13} \mathrm{C}$ NMR $\left(\mathrm{CDCl}_{3}\right) \delta 155.67 .153 .49,1.35 .13,134.25 .133 .31 .130 .77$. 129.56. 126.14. 123.87. 123.35 and 99.07 (Ar). 73.59. 71.51. 70.75 and $57.25\left(-\mathrm{OCH}_{2}\right)$ ). 31.41 and $27.17\left(\mathrm{ArCH}_{2} \mathrm{Ar}\right), 3 \mathrm{a}: \mathrm{mp}-$ 250-252 'C ' ${ }^{\prime}$ NMR (CDCl $) \delta 7.21-7.18$ (d ol d. 4H. ArH. $J-$ 7.29 II7). 7.16-7.1.3 (d of d. 4H. ArHI. $J-7.62$ HJ). 7.02 (t. 4 H. $\Lambda \mathrm{rII}, J-7.44 \mathrm{II}$ ), 6.69 (t. 2II, ArHI. $J-7.47 \mathrm{HI}$ ). 6.52 (d. 4II, $\wedge \mathrm{rI}, J-6.62 \mathrm{II}$ ), 5.04 (s. $4 \mathrm{HI} .-\left(\mathrm{CCH}_{2}-\right), 4.56$ (d. $4 \mathrm{II}, \mathrm{ArCII}_{2} \wedge \mathrm{r} . J$ $-15.3 \mathrm{~Hz} . s m) .4 .01$ (s. $4 \mathrm{H} . \mathrm{ArCH} \mathrm{Ar} a m i) .3 .65$ (s. $\left.6 \mathrm{H} .-\mathrm{OCH}_{3}\right)$. $3.58-3.54$ (m. 6H. $-\left(\mathrm{CCH}_{2} \mathrm{CH}_{2} \mathrm{O}-\right.$ ) $.3 .49-3.41$ (m. $10 \mathrm{H} . \mathrm{ArCH}_{2} \mathrm{Ar}$ and $-\left(\mathrm{CH}_{2} \mathrm{CH}_{2} \mathrm{O}-\right) .3 .23$ (m. $\left.4 \mathrm{H} .-\mathrm{OCH}_{2} \mathrm{CH}_{2} \mathrm{O}-\right) .3 .11$ (m. $4 \mathrm{H}$. $\left.-0 \mathrm{CII}_{2} \mathrm{CII}_{2} \mathrm{O}-\right) .3 .02\left(\mathrm{~m}, 4 \mathrm{II},-\mathrm{OCH} \mathrm{CH}_{2} \mathrm{CH} \mathrm{O}_{2} \mathrm{O}\right) .{ }^{12} \mathrm{C}$ NMR $\left(\mathrm{CDCl}_{3}\right) \delta$ 156.42. 152.92. 1.34.65, 1.33.96, 1.33.62, 1.30.2.3. 129.74. 126.60. 124.00. 123.86. 123.47 and $99.82(\mathrm{Ar}) .72 .72 .70 .85,70.06$ and $57.67\left(-\mathrm{CCH}_{2}-\right) .31 .77$ and $31.55\left(\mathrm{ArCH}_{2} \mathrm{Ar}\right), 2 \mathrm{~b}: \mathrm{mp}-234-236$ "C: ${ }^{1} \mathrm{H} \mathrm{NMR}\left(\mathrm{CDCl}_{3}\right) 87.63$ (d. $\left.4 \mathrm{H} . \mathrm{ArH} . J-6.72 \mathrm{~Hz}\right) .7 .55$ (c. 4H. ArH. $J-4.62 \mathrm{~Hz}$ ). 7.43-7.34 (m. 6H. ArH). 7.09-7.07 (d ol t. $8 \mathrm{II}, \wedge \mathrm{rI}$ ), 6.33 (t. 2HI. ArII, $J-7.65 \mathrm{I}$ (7), 6.09 (d, $4 \mathrm{II}$, Arl $J-$


$16.2 \mathrm{HJ}$ ). 4.60 (d. 2II, $\mathrm{ArCH} \wedge \mathrm{Ar}, J-1.3 .32 \mathrm{I}$ (7). 3.99 (t. $4 \mathrm{II}$, $-\left(\mathrm{CCH}_{2} \mathrm{CH}_{2} \mathrm{O}-. . J-8.73 \mathrm{~Hz}\right), 3.83-3.69\left(\mathrm{~m} .12 \mathrm{H} .-\mathrm{OCH}_{2} \mathrm{CH}_{2} \mathrm{O}-\right)_{-}$). $3.54-3.38\left(\mathrm{~m} .10 \mathrm{H} . \mathrm{ArCH} \mathrm{H}_{2} \mathrm{Ar}\right.$ and $-\mathrm{OCH}_{2} \mathrm{CH}_{2} \mathrm{O}-$ ) $3.18(\mathrm{~d} .4 \mathrm{H}$. $-\left(\mathrm{CCH}_{2} \mathrm{CH}_{2} \mathrm{O}-. j-10.74 \mathrm{~Hz}\right),{ }^{17} \mathrm{C}$ NMR $\left(\mathrm{CDCl}_{3}\right) \delta 155.68$. $154.00,138.16,1.35 .15,1.34 .37,133.28,1.30 .70 .129 .53 .128 .40$. $127.67,126.17,123.81$ and $123.21(\wedge \mathrm{r}) .73 .59 .73 .49,71.47$ and $70.68\left(-\mathrm{OCI}_{2}\right), 30.87$ and $27.14\left(\mathrm{ArCH}_{2} \Lambda_{\mathrm{r}}\right)+\mathrm{t} \mathrm{mp}-190{ }^{\circ} \mathrm{C}$ dec: ${ }^{1} \mathrm{H}$ NMR $\left(\mathrm{CDCl}_{3}\right.$ ) 87.72 (s. $\left.2 \mathrm{H} . \mathrm{OH}\right) .7 .03$ (br. s. $4 \mathrm{H} . \mathrm{ArH}$ ). 6.86 (br. s. 4H. ArH). 6.77 (t. $2 \mathrm{H}$. ArH. $.7-7.44 \mathrm{~Hz}$ ). 6.59 (br. s. 8H. ArH). 4.10 (m. 14H. ArCH Ar and $-\mathrm{OCH}_{2} \mathrm{CH}_{2} \mathrm{O}$-) $3.80(\mathrm{~m}$. $\left.11 \mathrm{IH},-\mathrm{OCI} \mathrm{CHI}_{2} \mathrm{O}-\right), 3.56\left(\mathrm{~m}, 11 \mathrm{HI}, \mathrm{ArCH}_{2} \mathrm{Ar}\right.$ and $\left.-\mathrm{OCI} \mathrm{Cl}_{2} \mathrm{CH}_{2} \mathrm{O}-\right)$. ${ }_{13}^{13} \mathrm{C}$ NMR $\left(\mathrm{CDCl}_{3}\right) \delta 154.36,152.35,13.79,133.27 .129 .08$. $128.68,128.39,128.19,124.57$ and $120.07(\mathrm{Ar}) .72 .85,70.27$ and $70.12\left(-\mathrm{OCH}_{2}-\right) .31 .67$ and $29.61\left(\mathrm{ArCH}_{2} \mathrm{Ar}\right)$. 